\title{
Implicit integration of simple breakage constitutive model for crushable granular materials: A numerical test
}

Chunshun Zhang**1,2 ${ }^{\text {Jian } \mathrm{Ji}^{2} \text {, Sheng-Qi Yang }}{ }^{1}$, and Jayantha Kodikara ${ }^{2}$

${ }^{1}$ State Key Laboratory for Geomechanics and Deep Underground Engineering, China University of Mining and Technology, Xuzhou 221116, PR China

${ }^{2}$ Department of Civil Engineering, Monash University, Clayton, Vic 3800, Australia

\begin{abstract}
In the context of the recently developed breakage mechanics that is based on thermodynamics principles, this paper presents a mathematical modelling procedure to implement the simple (i.e., linear elastoplastic) breakage constitutive model using finite element analysis (FEA) with illustrations by engineering applications. More informative mathematical derivation procedures of energy dissipations, plastic potential, yield function and non-associated flow rules are presented. In contrast, the existing relevant publications often lack sufficient elaboration, leaving knowledge gaps in the full understanding the model. This is followed by a series of numerical simulations in ABAQUS to test the model at the constitutive level. Various isotropic and triaxial shear tests in drained or undrained conditions are tested to illustrate the key features of the breakage model, which seem to be overlooked in the literature. Finally, a few numerical results are compared with experimental shear tests to demonstrate the ability of the simple breakage model in reflecting mechanical responses of crushable granular aggregates.
\end{abstract}

Keywords: breakage mechanics; simple breakage model; backward Euler return algorithm; FEA; granular materials 


\section{Introduction}

Different stress return algorithms have been proposed to integrate the constitutive relationships to compute the increments in stresses and state variables. One of the relatively easy algorithms is the so-called 'explicit scheme'. This integration scheme enables the updated quantities at time $t+\Delta t$ to be calculated based on known quantities at time $t$. Its notable advantage is the simplicity in FE implementation and it has been widely used in geomechanics [1]. However, the yield condition is not guaranteed at time $t+\Delta t$ in such a forward integration process. As a result, the calculated quantities, for instance the plastic multiplier at time $t+\Delta t$, is not satisfied in the yield condition. This causes the solution over many increments to drift away from the yielding surface [2]. Moreover, the time step size, $\Delta t$, cannot be too large. Otherwise, incorrect results will be encountered. Therefore, the application of explicit schemes is usually limited to some simple constitutive models (e.g., linear elastic model). For complex nonlinear constitutive models, the explicit scheme is usually neither efficient nor applicable despite that some researchers have been attempting to improve the performance of the explicit scheme. A good example is the explicit method with automatic substepping and error control as proposed by [3] for a suction-dependent unsaturated soil model, and more recently by [4] for integrating the well-known Barcelona Basic Model.

In contrast to the explicit scheme, another integration algorithm is called 'fully implicit scheme'. In detail, an (elastic) trial stress increment is firstly computed to obtain the updated trial stress $\sigma_{t+\Delta t}^{t r}$. The trial stress will be outside the yield surface if the yield condition is not satisfied. On this occasion, the trial stress is then brought back onto the yield surface at time $t+\Delta t$ with a plastic correction. This is known as stress return process that must be solved iteratively, of which a widely used method is the Newton method. Fully implicit method ensures the yield condition that is satisfied at each time increment, thus avoiding the deviation from the yield surface, as commonly encountered in the explicit scheme. In addition, the fully implicit scheme allows for the use of significantly larger time increments, which can give rise to faster solutions [2]. Note that the fully implicit method has been widely used in geomechanics [5-7] and general complex elastoplastic and viscoplastic materials , and 
models of which the mechanical responses are determined by all principal stress invariants .

The constitutive equations can also be integrated using a backward Euler return algorithm [6, 8-14]. This might be considered to be a 'semi-implicit' method with first-order accuracy, as it still follows the idea of stress return but relies on the firstorder of Taylor's expansion of the yield function. It is much simpler than the fully implicit method regarding the stress return process because the iteration is not strictly needed to calculate the updated stresses. Therefore the semi-implicit method makes the FE implementation much easier compared with the fully implicit method. Moreover, the semi-implicit method enables quantities to be updated almost as accurately as those obtained from the fully implicit method. Due to its simplicity, stability and high level of accuracy, the semi-implicit integration method is used in this paper to implement the simple breakage model.

The objective of this paper is to numerically verify the implementation of the simple breakage model. To achieve this, fundamental features of the breakage model and its thermodynamically consistent constitutive equations are briefly reviewed in Section 2. Especially, the relevant issues in model derivations of the energy dissipations, plastic potential, yield function and non-associated flow rules are elaborated to avoid incompleteness of understanding, as often encountered in the existing literatures. Section 3 illustrates the FE implementation by a standard return mapping method. This shows the model's versatility in various numerical applications. Section 4 tests the efficiency of the FE implementation at the material point level under various conventional loading conditions. This helps to understand the fundamental features of the model, which seem to be overlooked in the literature. Then in Section 5, laboratory testing of some weak to hard granular materials are numerically modelled to demonstrate some simple application, where the experimental data are in good agreement with the numerical counterparts. Summaries and discussions are presented in the last section.

\section{Discussion of the breakage mechanics and simple breakage model}

The significance of thermodynamics in the formulation and development of 
constitutive models has been widely acknowledged [15-18]. A rigorous constitutive model must satisfy both the first and second laws of thermodynamics in order to be consistent and physically meaningful. Otherwise, a model that does not obey the thermodynamics framework may not be used confidently in describing a material behaviour [16]. The importance of obeying thermodynamics framework in developing constitutive models has been recently stressed by Al-Rub and Darabi [19] and Darabi et al. [20], who established a general thermodynamically consistent framework for coupling various mechanisms such as temperature, viscoelasticity, viscoplasticity, viscodamage, and micro-damage healing for constitutive modeling of time- and ratedependent materials.

On the other hand, the formulation of continuum breakage mechanics and simple breakage model $[21,22]$ were developed on the basis of thermodynamics principles. Following the thermodynamics principles, the breakage mechanics emphasises the significance of linking micro to macro scales (i.e., avoiding as much as possible the arbitrary mathematical structures) in constitutive modelling by incorporating grain size distribution (GSD) and its evolution (through breakage, B) to capture the macroscopic soil behaviour. In this way, the underlying microscopic process connects with macroscopic behaviour, which many other continuum theories fail to capture.

There are various thermodynamically consistent breakage models that have been derived from the breakage mechanics, providing physical explanations for many of the phenomenological aspects of crushable soil behaviour. Thus, these models have far been used in different engineering applications, including geophysics [23, 24], rock mechanics [25], foundation engineering [26], unsaturated soil mechanics [27], and a general breakage model accounting for finite deformation and porous compaction and dilation [28]. Among those breakage models, the focus of the present study is the simple breakage model that is the simplest and fundamental form among all other relatively more complicated breakage models. According to the simple breakage model, the macroscopic specific elastic strain energy stored in a granular aggregate including particles of various sizes is simplified using linear elasticity [29]:

$$
\Psi=(1-\vartheta B)\left(\frac{1}{2} K \varepsilon_{v}^{e^{2}}+\frac{3}{2} G \varepsilon_{s}^{e^{2}}\right)
$$


where,

- $K$ and $G$ respectively represent the bulk and shear moduli;

- $\varepsilon_{v}^{e}$ and $\varepsilon_{s}^{e}$ are the elastic volumetric and shear strains in triaxial conditions, respectively;

- $\vartheta$ (from 0 to 1$)$ is a grading index which indicates the distance between the initial and ultimate GSD's [21];

- $B$ (from 0 to 1 ) is a breakage index, an internal state variable in a thermodynamic sense. $B$ can be used to connect the current cumulative GSD (by mass), $F(x)$, with the initial cumulative $\mathrm{GSD}, F_{0}(x)$, and ultimate cumulative GSD, $F_{\mathrm{u}}(x)$ based on the grain sizes $x$, by a linear relationship [21]:

$F(x, B)=(1-B) F_{0}(x)+B F_{u}(x)$

\section{Constitutive equations}

From the framework of thermodynamics [21, 22, 24], constitutive equations are defined by degrading elasticity equations as follows:

$$
\begin{aligned}
& p=\frac{\partial \Psi}{\partial \varepsilon_{v}^{e}}=(1-\vartheta B) K \varepsilon_{v}^{e} \\
& q=\frac{\partial \Psi}{\partial \varepsilon_{s}^{e}}=3(1-\vartheta B) G \varepsilon_{s}^{e} \\
& E_{B}=-\frac{\partial \Psi}{\partial B}=\frac{\vartheta}{2}\left(K \varepsilon_{v}^{e^{2}}+3 G \varepsilon_{s}^{e^{2}}\right)=\frac{\vartheta}{2(1-\vartheta B)^{2}}\left(\frac{p^{2}}{K}+\frac{q^{2}}{3 G}\right)
\end{aligned}
$$

where,

- $\quad p$ and $q$ respectively represent the mean and triaxial shear stresses;

- $E_{B}$ is a breakage energy, a stress-like conjugate that describes the total stored free energy available for release from the granular aggregate fractured from the initial to ultimate states of GSD.

\section{Energy dissipations}

Energy dissipations describe energy losses of the granular materials during crushing process, which are accomplished through a rational mathematical formulation. The mathematical equations of energy dissipations have been presented and adopted by many existing relevant works $[23,24,26]$ without sufficient details regarding their mathematical formulations. The following derivations are therefore presented to show their rationality in the formulations. 
Breakage mechanics identifies three sources of energy dissipations of particle crushing in granular materials, i.e., particle breakage, plastic volumetric and shear strains (or deformations). In conventional rate-independent plasticity theory, the increments of plastic dissipations are assumed to be homogeneous first order functions of the plastic volumetric and shear strain increments, respectively:

$$
\begin{aligned}
& \delta \Phi_{p}^{v}=\frac{\partial \delta \Phi_{p}^{v}}{\partial \delta \varepsilon_{v}^{p}} \delta \varepsilon_{v}^{p} \\
& \delta \Phi_{p}^{s}=\frac{\partial \delta \Phi_{p}^{s}}{\partial \delta \varepsilon_{s}^{p}} \delta \varepsilon_{s}^{p}
\end{aligned}
$$

where $\delta \Phi_{p}^{v}$ and $\delta \Phi_{p}^{s}$ denote the increments of plastic volumetric and shear dissipations via the change of incremental volumetric and shear strains, $\delta \varepsilon_{v}^{p}$ and $\delta \varepsilon_{s}^{p}$.

Analogous to the above formulations, the increment of breakage dissipation, $\delta \Phi_{B}$, is also assumed to take a first order homogeneous function [21]:

$$
\delta \Phi_{B}=\frac{\partial \delta \Phi_{B}}{\partial \delta B} \delta B
$$

where $\delta \Phi_{B}$ denotes the increment of breakage dissipation via the change of incremental breakage, $\delta B$.

On the other hand, the $p, q$ and $E_{B}$ can also be obtained from dissipation potential (in incremental form), $\delta \Phi$, based on the energy conservation equation [21]:

$$
\begin{gathered}
p=\frac{\partial \delta \Phi}{\partial \delta \varepsilon_{v}^{p}} \\
q=\frac{\partial \delta \Phi}{\partial \delta \varepsilon_{s}^{p}} \\
E_{B}=\frac{\partial \delta \Phi}{\partial \delta B}
\end{gathered}
$$

In extreme cases where the energy loss is exclusively induced either by plastic volumetric, shear dissipation or breakage dissipation, Eqns. (9) to (11) become:

$$
p=\frac{\partial \delta \Phi_{p}^{v}}{\partial \delta \varepsilon_{v}^{p}}
$$




$$
\begin{gathered}
q=\frac{\partial \delta \Phi_{p}^{s}}{\partial \delta \varepsilon_{s}^{p}} \\
E_{B}=\frac{\partial \delta \Phi_{B}}{\partial \delta B}
\end{gathered}
$$

Substituting Eqns. (12)-(14) into Eqns. (6)-(8), we have:

$$
\begin{aligned}
& \delta \Phi_{p}^{v}=p \delta \varepsilon_{v}^{p} \\
& \delta \Phi_{p}^{s}=q \delta \varepsilon_{s}^{p} \\
& \delta \Phi_{B}=E_{B} \delta B
\end{aligned}
$$

In more general cases where the energy loss is a combination of the result from the three sources, a few modifications to Eqns. (15)-(17) are manipulated as follows:

- Under isotropic compression conditions, $\delta \Phi_{p}^{v}$ induced by particlerearrangement passively arises from particle breakage, $\delta \Phi_{B}$ and then the both dissipation mechanisms compete (couple) with each other during fracture of granules. A coupling angle, $\omega$ (between $0^{\circ}$ and $90^{\circ}$ ) is therefore introduced to describe the devotions between $\delta \Phi_{p}^{v}$ and $\delta \Phi_{B}$ [22]. Under shearing conditions, the induced dissipation $\delta \Phi_{p}^{s}$ is derived on the basis of Coulomb's friction law.

- A mathematically favourable expressions of $\delta \Phi_{p}^{v}$ and $\delta \Phi_{B}$ can be formulated by incorporating the breakage-yield criterion, $E_{B}(1-B)^{2} / E_{c}=1$ [21]. Here $E_{c}$ is termed "breakage energy constant" obtained from critical isotropic confining pressure $p_{c}$ to initialise grain crushing of the aggregate via $p_{c}=\sqrt{2 K E_{c} / \vartheta}$ [30]

Applying the above considerations to Eqns. (15)-(17) leads to:

$$
\begin{aligned}
& \delta \Phi_{B}=\frac{\sqrt{E_{B} E_{c}}}{(1-B) \cos \omega} \delta B \\
& \delta \Phi_{p}^{v}=\frac{p}{(1-B) \sin \omega} \sqrt{\frac{E_{c}}{E_{B}}} \delta \varepsilon_{v}^{p} \\
& \delta \Phi_{p}^{s}=M p\left|\delta \varepsilon_{s}^{p}\right|
\end{aligned}
$$

where $M=q_{f} / p_{f}$, is the ratio of shear stress to the volumetric one at failure. 


\section{Plastic potential}

Plastic potential $y^{*}$, or termed yield function in dissipative triaxial stress/breakage space, has been adopted by $[23,24,26]$. However, the detailed derivation has not been fully presented, leading to an incomplete understanding of the breakage mechanics and simple breakage model. In the following, we provide concise but essential steps to derive $y^{*}$ based on increment of dissipation potential $\delta \Phi$ that associates with $\delta \Phi_{p}^{v}, \delta \Phi_{p}^{s}$ and $\delta \Phi_{B}$.

In breakage mechanics, $\delta \Phi$ is a combination of coupling $\delta \Phi_{p}^{v}, \delta \Phi_{p}^{s}$ and $\delta \Phi_{B}$ together in a convenient form [24]:

$$
\delta \Phi=\sqrt{\delta \Phi_{B}^{2}+\delta \Phi_{p}^{v^{2}}+\delta \Phi_{p}^{s^{2}}}=\sqrt{\left(\Delta_{1} \delta B\right)^{2}+\left(\Delta_{2} \delta \varepsilon_{v}^{p}\right)^{2}+\left(\Delta_{3} \delta \varepsilon_{s}^{p}\right)^{2}}
$$

where recalling Eqns (18)-(20), we have:

$$
\begin{aligned}
& \Delta_{1}=\frac{\partial \delta \Phi_{B}}{\partial \delta B}=\frac{\sqrt{E_{B} E_{c}}}{(1-B) \cos \omega} \\
& \Delta_{2}=\frac{\partial \delta \Phi_{p}^{v}}{\partial \delta \varepsilon_{v}^{p}}=\frac{p}{(1-B) \sin \omega} \\
& \Delta_{3}=\frac{\partial \delta \Phi_{p}^{s}}{\partial \delta \varepsilon_{s}^{p}}=M p
\end{aligned}
$$

Eq. (21) can be rearranged as below:

$$
\begin{aligned}
\delta \Phi & =\frac{\partial \delta \Phi}{\partial \delta \Phi_{B}} \delta \Phi_{B}+\frac{\partial \delta \Phi}{\partial \delta \Phi_{p}^{v}} \delta \Phi_{p}^{v}+\frac{\partial \delta \Phi}{\partial \delta \Phi_{p}^{s}} \delta \Phi_{p}^{s} \\
& =\frac{\partial \delta \Phi}{\partial \delta \Phi_{B}} \frac{\partial \delta \Phi_{B}}{\partial \delta B} \delta B+\frac{\partial \delta \Phi}{\partial \delta \Phi_{p}^{v}} \frac{\partial \delta \Phi_{p}^{v}}{\partial \delta \varepsilon_{v}^{p}} \delta \varepsilon_{v}^{p}+\frac{\partial \delta \Phi}{\partial \delta \Phi_{p}^{s}} \frac{\partial \delta \Phi_{p}^{s}}{\partial \delta \varepsilon_{s}^{p}} \delta \varepsilon_{s}^{p} \\
& =\frac{\partial \delta \Phi}{\partial \delta B} \delta B+\frac{\partial \delta \Phi}{\partial \delta \varepsilon_{v}^{p}} \delta \varepsilon_{v}^{p}+\frac{\partial \delta \Phi}{\partial \delta \varepsilon_{s}^{p}} \delta \varepsilon_{s}^{p}
\end{aligned}
$$

Eq. (25) proves that Eq. (21) is a first order homogenous function.

From Eq. (21), we have:

$$
\begin{aligned}
& \frac{\partial \delta \Phi}{\partial \delta B}=\frac{\Delta_{1}^{2}}{\delta \Phi} \delta B \\
& \frac{\partial \delta \Phi}{\partial \delta \varepsilon_{v}^{p}}=\frac{\Delta_{2}{ }^{2}}{\delta \Phi} \delta \varepsilon_{v}^{p}
\end{aligned}
$$




$$
\frac{\partial \delta \Phi}{\partial \delta \varepsilon_{s}^{p}}=\frac{\Delta_{3}^{2}}{\delta \Phi} \delta \varepsilon_{s}^{p}
$$

Using Eqns. (26)-(28), the Eq. (25) becomes:

$$
\delta \Phi=\left(\frac{\partial \delta \Phi}{\partial \delta B}\right)^{2} \frac{\delta \Phi}{\Delta_{1}^{2}}+\left(\frac{\partial \delta \Phi}{\partial \delta \varepsilon_{v}^{p}}\right)^{2} \frac{\delta \Phi}{\Delta_{2}{ }^{2}}+\left(\frac{\partial \delta \Phi}{\partial \delta \varepsilon_{s}^{p}}\right)^{2} \frac{\delta \Phi}{\Delta_{3}^{2}}
$$

Based on Eq. (32), the plastic potential $y^{*}$ can be expressed as:

$$
y^{*}=\left(\frac{\partial \delta \Phi / \partial \delta B}{\partial \delta \Phi_{B} / \partial \delta B}\right)^{2}+\left(\frac{\partial \delta \Phi / \partial \delta \varepsilon_{v}^{p}}{\partial \delta \Phi_{p}^{v} / \partial \delta \varepsilon_{v}^{p}}\right)^{2}+\left(\frac{\partial \delta \Phi / \partial \delta \varepsilon_{s}^{p}}{\partial \delta \Phi_{p}^{s} / \partial \delta \varepsilon_{s}^{p}}\right)^{2}-1
$$

Recalling Eqns. (9)-(11), $y^{*}$ can be replaced by:

$$
y^{*}=\left(\frac{E_{B}}{\partial \delta \Phi_{B} / \partial \delta B}\right)^{2}+\left(\frac{p}{\partial \delta \Phi_{p}^{v} / \partial \delta \varepsilon_{v}^{p}}\right)^{2}+\left(\frac{q}{\partial \delta \Phi_{p}^{s} / \partial \delta \varepsilon_{s}^{p}}\right)^{2}-1
$$

\section{Non-associated flow rules}

The non-associated flow rules are derived from the $y^{*}$ with the help of Eqns. (18)-(20):

$$
\begin{aligned}
& \delta \varepsilon_{v}^{p}=\delta \lambda \frac{\partial y^{*}}{\partial p}=\delta \lambda \frac{2 E_{B}(1-B)^{2} \sin ^{2} \omega}{p E_{c}} \\
& \delta \varepsilon_{s}^{p}=\delta \lambda \frac{\partial y^{*}}{\partial q}=\delta \lambda \frac{2 q}{(M p)^{2}} \\
& \delta B=\delta \lambda \frac{\partial y^{*}}{\partial E_{B}}=\delta \lambda \frac{2(1-B)^{2} \cos ^{2} \omega}{E_{c}}
\end{aligned}
$$

where, $\delta \lambda$ is a non-negative multiplier. The plastic-breakage coupling angle coupling angle $\omega$ governs the energy consumption due to $\delta \varepsilon_{v}^{p}$ and $\delta B . \omega=0^{\circ}$ indicates that grain crushing completely dominates the energy loss, and otherwise, $\omega=90^{\circ}$ indicates plastic volumetric strain completely dominates the energy loss in isotropic compression conditions.

\section{Yield function}

Substituting Eqns. (18)-(20) into (31), $y^{*}$ can be replaced by elastic-plastic-breakage yield function, $y$ in the mixed stress/breakage space [29, 30]:

$$
y=\frac{E_{B}}{E_{c}}(1-B)^{2}+\left(\frac{q}{M p}\right)^{2}-1 \leq 0
$$

Note that although $y$ is derived from $y^{*}$, they are not the same for the purpose of 
differentiation. It is easy to prove that $\partial y^{*} / \partial p \neq \partial y / \partial p$ and $\partial y^{*} / \partial E_{B} \neq \partial y / \partial E_{B}$. An illustration to show the non-associated flow rules of the simple breakage model has been presented in [31].

As a summary of the above simple breakage model, it contains only five physically identifiable mechanical parameters: shear and bulk modulus $G$ and $K$, friction coefficient $M$, critical breakage energy constant $E_{c}$, and coupling angle $\omega$ between the friction and breakage dissipative processes. All of these parameters can be measured and calibrated using a single standard triaxial test involving a stage of isotropic compression followed by a stage of drained or undrained shear. In addition, the model responds to variations in the initial GSD through the grading (physical) index $\vartheta$.

\section{Numerical implementation in FEA}

In order to implement the simple elastic breakage constitutive model in FEA, we will describe herein the triaxial model in its tensorial form. Note the sign convention follows that of solid mechanics, where compression is negative for the implementation in ABAQUS. The triaxial stress and strain invariants read:

$$
\begin{aligned}
& p=-\frac{1}{3} \sigma_{k k} \\
& q=\sqrt{\frac{3}{2} s_{i j} s_{i j}} \\
& \varepsilon_{v}=-\varepsilon_{k k} \\
& \varepsilon_{s}=\sqrt{\frac{2}{3} e_{i j} e_{i j}}
\end{aligned}
$$

where, $\delta_{i j}$ is the Kronecker delta, and

$$
\begin{aligned}
& s_{i j}=\sigma_{i j}-\frac{\sigma_{k k}}{3} \delta_{i j} \\
& e_{i j}=\varepsilon_{i j}-\frac{\varepsilon_{k k}}{3} \delta_{i j}
\end{aligned}
$$

The elastic component of the constitutive model as previously shown by Eqns. (3-5) can be rewritten in tensorial form, such that: 
$\sigma_{i j}=(1-\vartheta B) D_{i j k l} \varepsilon_{k l}^{e}$

As can be seen, $(1-\vartheta B)$ acts as a scalar degradation function of linear elastic equation, where the linear elastic stiffness matrix $D_{i j k l}$ remains:

$$
D_{i j k l}=\left[\begin{array}{cccccc}
K+\frac{4}{3} G & K-\frac{2}{3} G & K-\frac{2}{3} G & 0 & 0 & 0 \\
K-\frac{2}{3} G & K+\frac{4}{3} G & K-\frac{2}{3} G & 0 & 0 & 0 \\
K-\frac{2}{3} G & K-\frac{2}{3} G & K+\frac{4}{3} G & 0 & 0 & 0 \\
0 & 0 & 0 & 2 G & 0 & 0 \\
0 & 0 & 0 & 0 & 2 G & 0 \\
0 & 0 & 0 & 0 & 0 & 2 G
\end{array}\right]
$$

Differentiating $y^{*}$ with respect to stresses, $\sigma_{i j}$ and the thermodynamic conjugate, $E_{B}$ results in the following non-associated flow rules in tensorial forms:

$$
\begin{aligned}
& \delta \varepsilon_{i j}^{p}=\delta \lambda\left(\frac{\partial y^{*}}{\partial p} \frac{\partial p}{\partial \sigma_{i j}}+\frac{\partial y^{*}}{\partial q} \frac{\partial q}{\partial \sigma_{i j}}\right)=\delta \lambda\left[-\frac{2(1-B)^{2} E_{B} \sin ^{2} \omega}{p E_{c}} \frac{\delta_{i j}}{3}+\frac{3 s_{i j}}{M^{2} p^{2}}\right]=\delta \lambda Q_{i j} \\
& \delta B=\delta \lambda \frac{\partial y^{*}}{\partial E_{B}}=\delta \lambda \frac{2(1-B)^{2} \cos ^{2} \omega}{E_{c}}=\delta \lambda R
\end{aligned}
$$

In order to compute the stress increment $\delta \sigma_{i j}$ based on the strain increment $\delta \varepsilon_{i j}$ obtained from the non-linear FEA at the current point $X$ (refer to Figure 1), first to consider the case where yielding occurs at a trial point $\mathrm{Y}$, i.e., $y^{\text {trial }}>0$. At this trial stress point, we assume a linear elastic behaviour with stiffness $(1-\vartheta B) D_{i j k l}$ for $\delta \varepsilon_{i j}$ resulting in a trial stress increment $\delta \sigma_{i j}^{\text {trial }}=(1-\vartheta B) D_{i j k l} \delta \varepsilon_{k l}$. Following first-order Taylor expansion, the yield function at the trial point Y (Figure 1) is written as:

$$
y=y^{\text {trial }}+\frac{\partial y}{\partial B} \delta B+\left(\frac{\partial y}{\partial p} \frac{\partial p}{\partial \sigma_{i j}}+\frac{\partial y}{\partial q} \frac{\partial q}{\partial \sigma_{i j}}\right) \delta \sigma_{i j}^{Y Z}=y^{\text {trial }}+\frac{\partial y}{\partial B} \delta B+M_{i j} \delta \sigma_{i j}^{Y Z}
$$




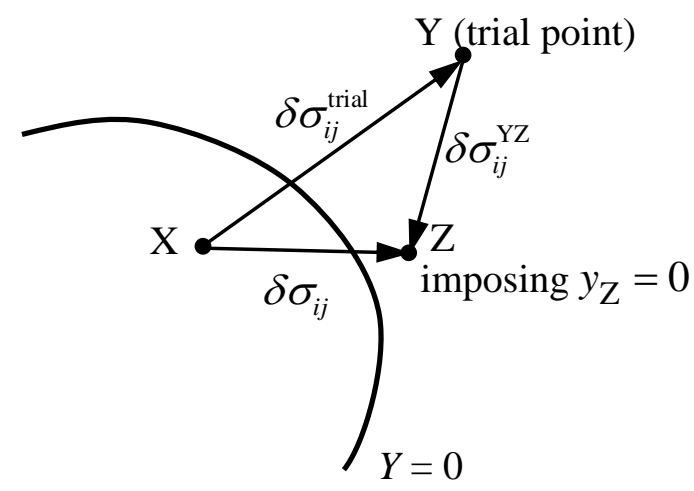

Figure 1 Schematics of the stress return algorithm.

The stress return (acts as a plastic corrector) from the points $Y$ to $Z$ (Figure 1), $\delta \sigma_{i j}^{\mathrm{YZ}}$ is computed by:

$$
\delta \sigma_{i j}^{Y Z}=-(1-\vartheta B) D_{i j k l} \delta \varepsilon_{k l}^{p}-\frac{\vartheta \sigma_{i j}}{1-\vartheta B} \delta B=-\delta \lambda\left[(1-\vartheta B) D_{i j k l} Q_{i j}+\frac{\vartheta \sigma_{i j}}{1-\vartheta B} R\right]
$$

Substituting Eq. (50) and the flow rule Eq. (48) into Eq. (49) results in:

$$
y=y^{\text {trial }}+\left[\frac{\partial y}{\partial B} R-M_{i j}\left((1-\vartheta B) D_{i j k l} Q_{k l}+\frac{\vartheta \sigma_{i j}}{1-\vartheta B} R\right)\right] \delta \lambda
$$

where $Q_{k l}$ and $R$ are defined in Eqns. (43-45). By doing so, the implication is that all variables and quantities in Eq. (46) are simply evaluated at a trial point Y. Imposing the yield condition at point $\mathrm{Z}, y=y_{\mathrm{Z}}=0$, we can obtain the multiplier $\delta \lambda$ as follows:

$$
\delta \lambda=\frac{-y^{\text {trial }}}{\frac{\partial y}{\partial B} R-M_{i j}\left((1-\vartheta B) D_{i j k l} Q_{k l}+\frac{\vartheta \sigma_{i j}}{1-\vartheta B} R\right)}=\frac{-y^{\text {trial }}}{N}
$$

At last, the total stress increment, $\delta \sigma_{i j}$ as the sum of $\delta \sigma_{i j}^{\text {trial }}$ and $\delta \sigma_{i j}^{\mathrm{YZ}}$ (Figure 1) is given as follows:

$$
\delta \sigma_{i j}=\delta \sigma_{i j}^{\mathrm{trial}}+\delta \sigma_{i j}^{\mathrm{YZ}}
$$

The above algorithm has been fully implemented in UMAT, a widely used platform of user subroutine embedded into the commercial finite element package ABAQUS in this paper. 
Note that due to the linearization (i.e., first-order Taylor expansion) of the yield function in Eq. (46), the returned stress point $Z$ usually does not exactly lie on the new yield surface, e.g., $y\left(\left.B\right|_{Z},\left.p\right|_{Z},\left.q\right|_{Z}\right) \neq 0$; however, sufficient accuracy can be achieved with a relatively large increment, like $\Delta \varepsilon_{s}=10^{-2}$, which will be demonstrated later in Section 4.5. Alternatively, repetition of the above process, e.g., linearizing the yield function at point $Z$ and then following the same algorithm is a simple way to reduce the gap between the stress point and the yield surface. Improvement to the accuracy can also be made by dividing the total strain increment $\mathrm{d} \varepsilon_{i j}$ into sub-increments, and applying the above algorithm to each sub-increment.

\section{Tangent stiffness matrix}

Implicit finite element analysis using the Newton-Raphson iterative techniques requires the formulation of the tangent stiffness matrix (Jacobian). This matrix is of importance to increase the convergence rate. The derivation of Jacobian is straightforward by starting from the stress-strain relationship (Eq. (42)) written in:

$$
\begin{aligned}
\delta \sigma_{i j} & =(1-\vartheta B) D_{i j k l}\left(\delta \varepsilon_{k l}-\delta \varepsilon_{k l}^{p}\right)-\vartheta D_{i j k l} \varepsilon_{k l}^{e} \delta B \\
& =(1-\vartheta B) D_{i j k l} \delta \varepsilon_{k l}-(1-\vartheta B) D_{i j k l} \delta \varepsilon_{k l}^{p}-\vartheta D_{i j k l} \varepsilon_{k l}^{e} \delta B
\end{aligned}
$$

Substituting Eqns. (47-48) into the above:

$$
\delta \sigma_{i j}=(1-\vartheta B) D_{i j k l} \delta \varepsilon_{k l}-(1-\vartheta B) D_{i j k l} \delta \lambda Q_{i j}-\vartheta D_{i j k l} \varepsilon_{k l}^{e} \delta \lambda R
$$

As opposed to Eq. (46) in implicit integration, consistency condition in explicit integration is generally obtained as:

$$
\delta y=\frac{\partial y}{\partial B} \delta B+\left(\frac{\partial y}{\partial p} \frac{\partial p}{\partial \sigma_{i j}}+\frac{\partial y}{\partial q} \frac{\partial q}{\partial \sigma_{i j}}\right) \delta \sigma_{i j}=\frac{\partial y}{\partial B} \delta B+M_{i j} \delta \sigma_{i j}
$$

Substituting Eq. (52) and flow rule Eq. (45) into Eq. (53), we have:

$$
\delta y=(1-\vartheta B) M_{i j} D_{i j k l} \delta \varepsilon_{k l}-\left(\frac{\partial y}{\partial B} R-M_{i j}\left((1-\vartheta B) D_{i j k l} Q_{k l}+\frac{\vartheta \sigma_{i j}}{1-\vartheta B} R\right)\right) \delta \lambda
$$

The plasticity/breakage multiplier is then:

$$
\delta \lambda=\frac{(1-\vartheta B) M_{i j} D_{i j k l} \delta \varepsilon_{k l}}{\frac{\partial y}{\partial B} R-M_{i j}\left((1-\vartheta B) D_{i j k l} Q_{k l}+\frac{\vartheta \sigma_{i j}}{1-\vartheta B} R\right)}=\frac{T_{k l} \delta \varepsilon_{k l}}{N}
$$


Placing Eq. (55) into Eq. (52), we obtain:

$$
\delta \sigma_{i j}=\left((1-\vartheta B) D_{i j m n}-(1-\vartheta B) D_{i j k l} Q_{k l} \frac{T_{m n}}{N}-R \vartheta D_{i j k l} \varepsilon_{k l}^{e} \frac{T_{m n}}{N}\right) \delta \varepsilon_{m n}
$$

$T_{m n}$ is defined in Eq. (55). Thus the tangent stiffness matrix is:

$$
X_{i j m n}=(1-\vartheta B) D_{i j m n}-(1-\vartheta B) D_{i j k l} Q_{k l} \frac{T_{m n}}{N}-R \vartheta D_{i j k l} \varepsilon_{k l}^{e} \frac{T_{m n}}{N}
$$

where, the used notations and derivatives are listed below:

$$
\begin{aligned}
& \frac{\partial y}{\partial B}=\frac{\vartheta(1-B)}{E_{c}(1-\vartheta B)^{2}}\left(\frac{p^{2}}{K}+\frac{q^{2}}{3 G}\right) \\
& \frac{\partial y}{\partial p}=\frac{p \vartheta}{K E_{c}}\left(\frac{1-B}{1-\vartheta B}\right)^{2}-\frac{2 q^{2}}{M^{2} p^{3}} \\
& \frac{\partial y}{\partial q}=\frac{q \vartheta}{3 G E_{c}}\left(\frac{1-B}{1-\vartheta B}\right)^{2}+\frac{2 q}{M^{2} p^{2}} \\
& \frac{\partial p}{\partial \sigma_{i j}}=-\frac{\delta_{i j}}{3} \\
& \frac{\partial q}{\partial \sigma_{i j}}=\frac{\partial q}{\partial s_{k l}} \frac{\partial s_{k l}}{\partial \sigma_{i j}}=\frac{3}{2} \frac{s_{i j}}{q} \\
& M_{i j}=\left(\frac{\partial y}{\partial p} \frac{\partial p}{\partial \sigma_{i j}}+\frac{\partial y}{\partial q} \frac{\partial q}{\partial \sigma_{i j}}\right) \\
& T_{k l}=(1-\vartheta B) M_{i j} D_{i j k l} \delta \varepsilon_{k l} \\
& N=\frac{\partial y}{\partial B} R-M_{i j}\left((1-\vartheta B) D_{i j k l} Q_{k l}+\frac{\vartheta \sigma_{i j}}{1-\vartheta B} R\right)
\end{aligned}
$$

As can be seen from the above process, the implementation of the breakage model follows a standard procedure of stress return algorithm as a result of the versatile model formulation that obeys the thermodynamics. Therefore, the model can be relatively simply implemented without adding extra numerical difficulty, and be readily used in various numerical applications in FEAs.

\section{Verification of implementations}

To verify the FE implementation, it is necessary to test the model's response at the 
material point level using a single reduced integrated axisymmetric element, under various loading paths. To do this, the following four numerical experiments were conducted and the results obtained were compared with solutions from the triaxial model in generalized $p-q-B$ space.

The schematics of the single-element model for all verification cases are shown in Figure 2, in which only a quarter of the whole is modelled; while the boundary conditions vary from case to case, and they are illustrated in each case. The parameters, if not specified, are from parameters as listed in Table 1 that are cited from [24].

Table 1 Model parameters for numerical experiments

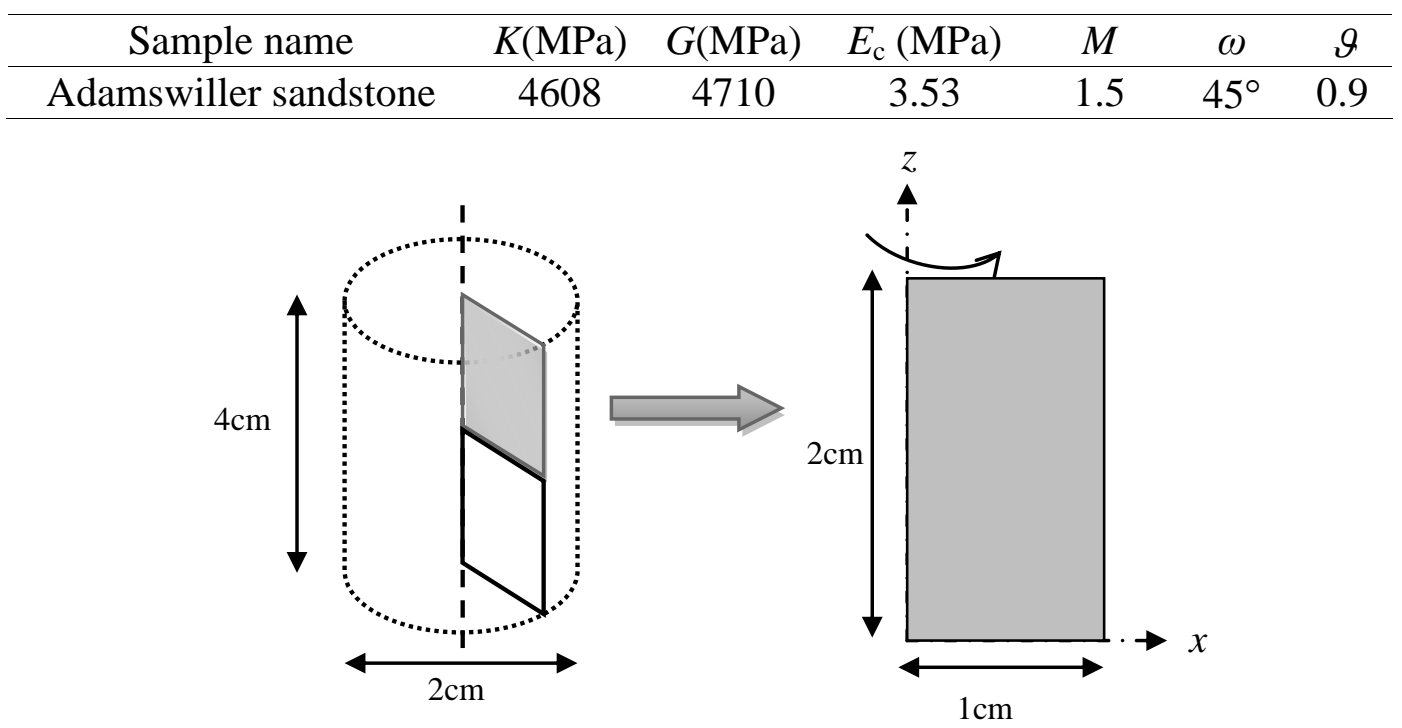

Figure 2 Axisymmetric finite element mesh (one element). Considering the geometry of the model, modelling of a quarter of the whole (the grey part) is sufficient.

\subsection{Isotropic loading condition}

As the simplest case, the isotropic loading condition is firstly applied to validate the implementation. The model's boundary conditions are shown in Figure 3. Increase confining pressure $p$ until a volumetric strain of $\varepsilon_{v}=0.5$. The model's mechanical responses are compared in Figure 4. 


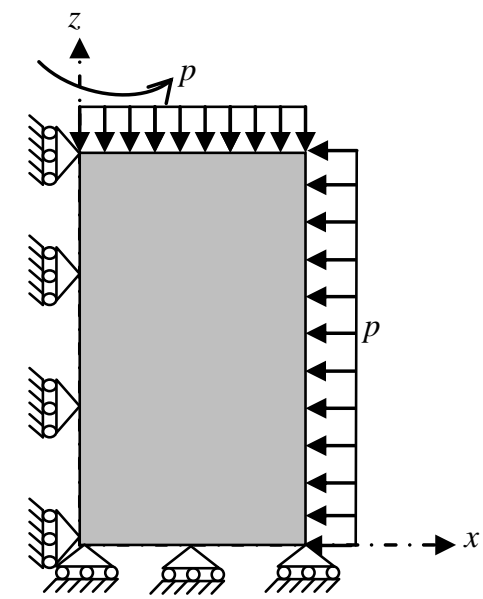

Figure 3 Boundary conditions of isotropic loaded specimen

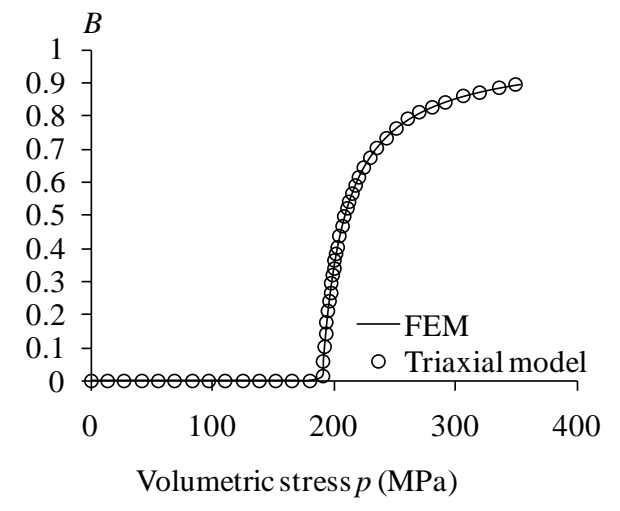

(a)

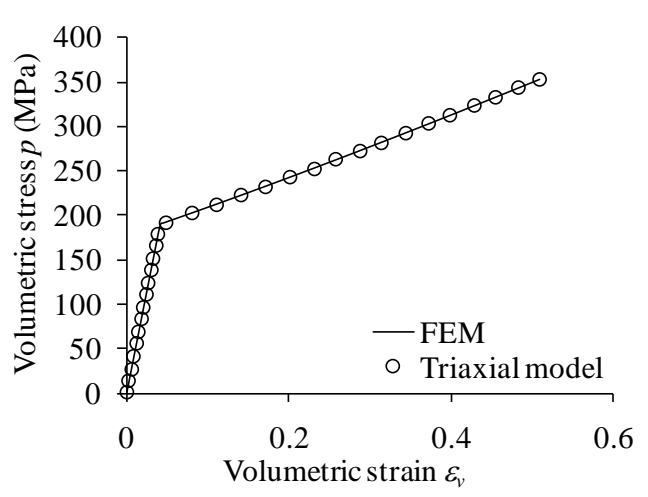

(b)

Figure 4 Validation of implementation in terms of (a) $p-B$ and (b) $p-\varepsilon_{v}$ relationship, under isotropic compression condition

\subsection{Isotropic loading-unloading-reloading condition}

While based on the same geometry used in Figure 3, in this case we firstly compress the sample until $\varepsilon_{v 1}=0.3$, then unload the model until $\varepsilon_{v 2}=0.15$ (here 'unloading' is realised by adding an increased isotropic tensile pressure), and finally recompress the model until $\varepsilon_{v 3}=0.6$. 


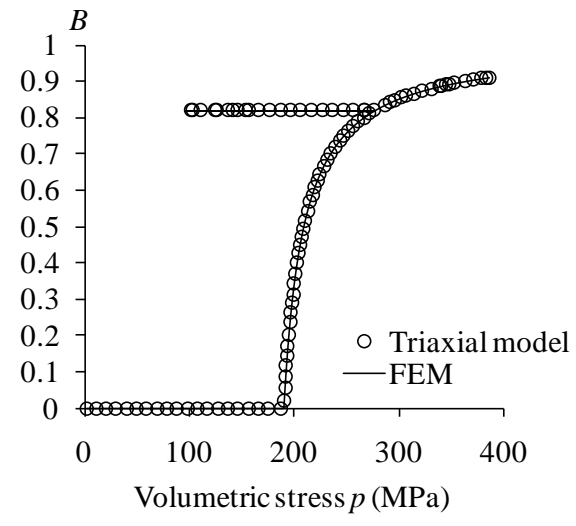

(a)

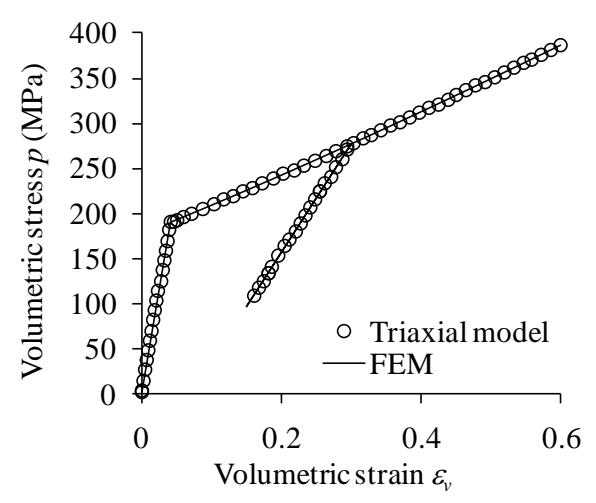

(b)

Figure 5 Validation of implementation in terms of (a) $p-B$ and (b) $p-\varepsilon_{v}$ relationship, under isotropic loading, unloading, and reloading conditions.

\subsection{Conventional drained loading with constant confining pressure}

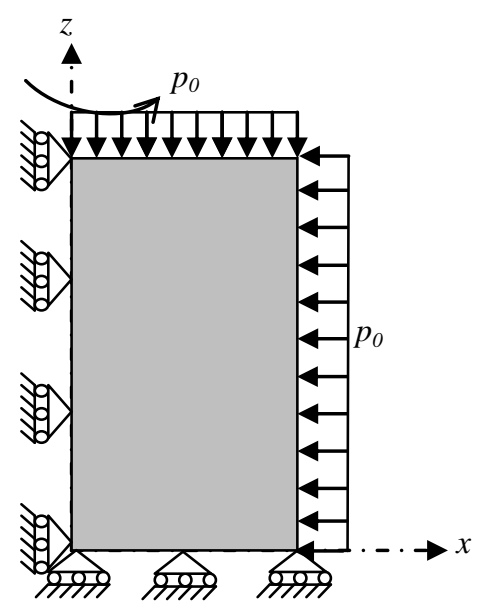

(a) Isotropic stage

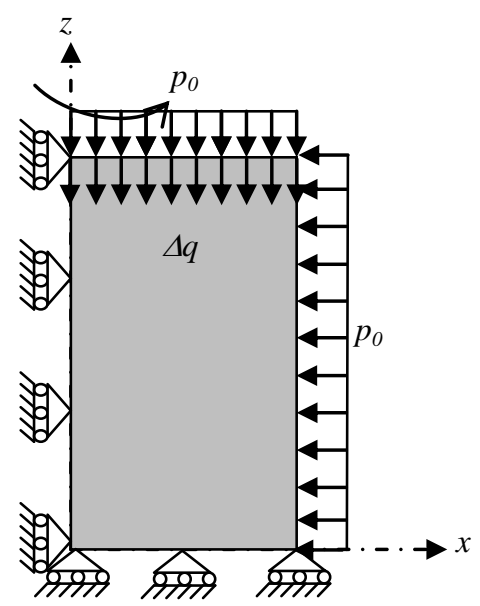

(b) Shear stage

Figure 6 Boundary conditions of conventional shear test

The boundary conditions for this case are illustrated in Figure 6, in which the model is firstly under an initial isotropic stress of $p_{0}=100 \mathrm{MPa}$. The model is then subjected to an increased shear stress $\Delta q$ till the shear strain $\varepsilon_{s}=0.3$. The model's responses to the triaxial model and finite element model (FEM) are compared in Figure 7.
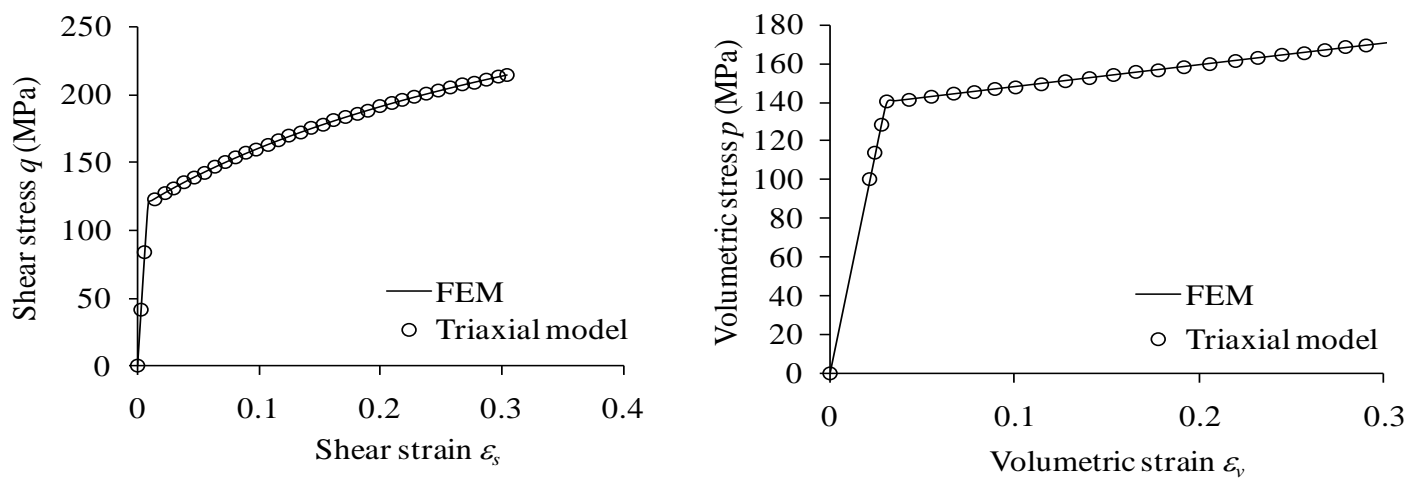
(a)

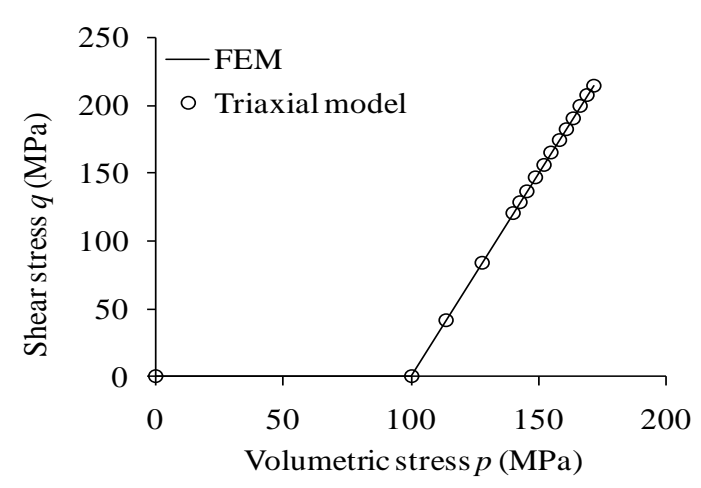

(c) (b)

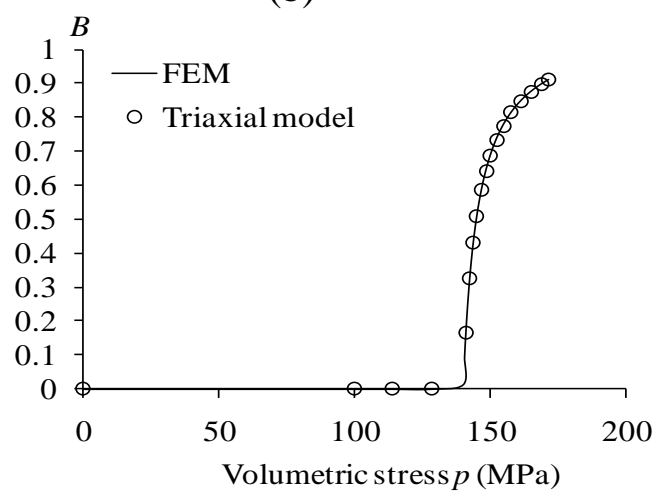

(d)

Figure 7 Validation of implementation in terms of (a) $q-\varepsilon_{s}$, (b) $p-\varepsilon_{v}$, (c) $p-q$ and (d) $p-B$ relationship, under conventional drained loading condition.

\subsection{Conventional undrained loading with constant confining pressure}

The FE undrained model is also based on Figure 6. To simulate the undrained behaviour of the soil model, all boundaries are impermeable. Therefore the permeability, $k$, can be defined arbitrarily. Let us take $k=0.002 \mathrm{~m} / \mathrm{s}$. Increase the shear stress $\Delta q$ till the $\varepsilon_{s}=1.0$. The results from the FEM and triaxial models are compared in Figure 8.

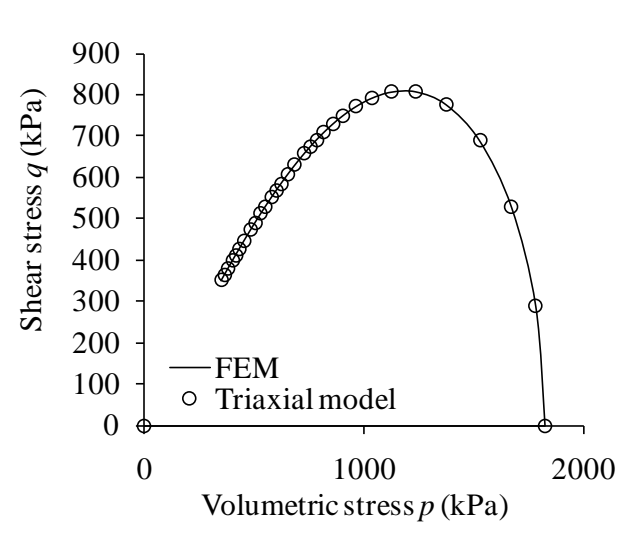

(a)

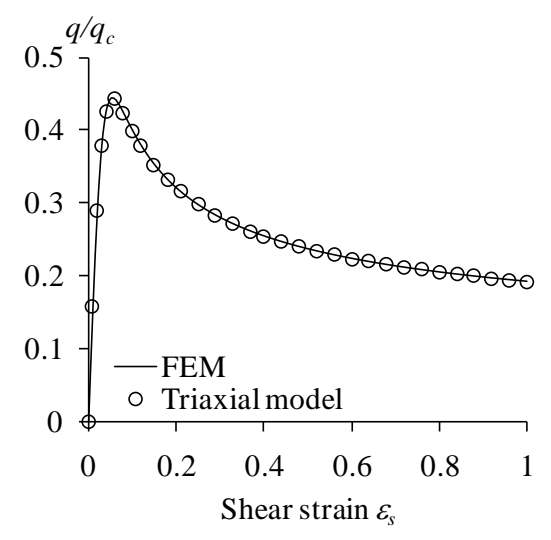

(b)

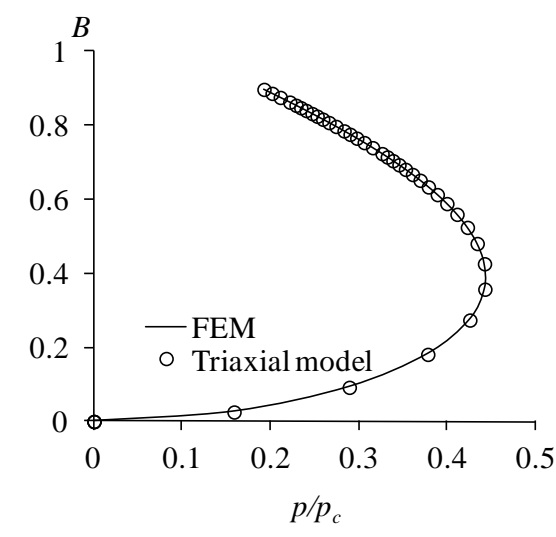


(c)

Figure 8 Validation of implementation in undrained loading condition in terms of (a) $p-q$, (b) normalized shear stress and shear strain relationship, $q_{t} p_{c}-\varepsilon_{s}$ and (c) normalized volumetric stress and breakage relationship, $p_{l} p_{c}-B$. Recall that $p_{c}=\sqrt{2 K E_{c} / \vartheta}$. The following parameters were used: $K=30000 \mathrm{kPa}, G=10000 \mathrm{kPa}, E_{c}=50 \mathrm{kPa}, M=1, \vartheta=0.9$ and $\omega=0^{\circ}$ (indicating no plastic volumetric strain).

\subsection{Accuracy verification}

To validate the accuracy of the implementation, different $\Delta \varepsilon_{s}$ (i.e. from $10^{-1}$ to $10^{-5}$ ) are selected for the conventional undrained test. As an example, only the results of $q_{/} p_{c^{-}} \varepsilon_{s}$ and $p_{l} p_{c}-B$ are plotted in Figure 9. It can be seen that good convergence is observed for $\Delta \varepsilon_{s}$ less or equal to $10^{-2}$. For $\Delta \varepsilon_{s}$ equal or less than $10^{-3}$, the results coincide with each other. Given $\Delta \varepsilon_{s}=10^{-3}$, the corresponding yielding value, $y$ (Eq. (12)) is about $10^{-4}$ that satisfies the requirement of numerical accuracy. Therefore, all simulations throughout this paper consider the both conditions of $\Delta \varepsilon_{S}=10^{-3}$ and $y=10^{-4}$ as the accuracy criterion.
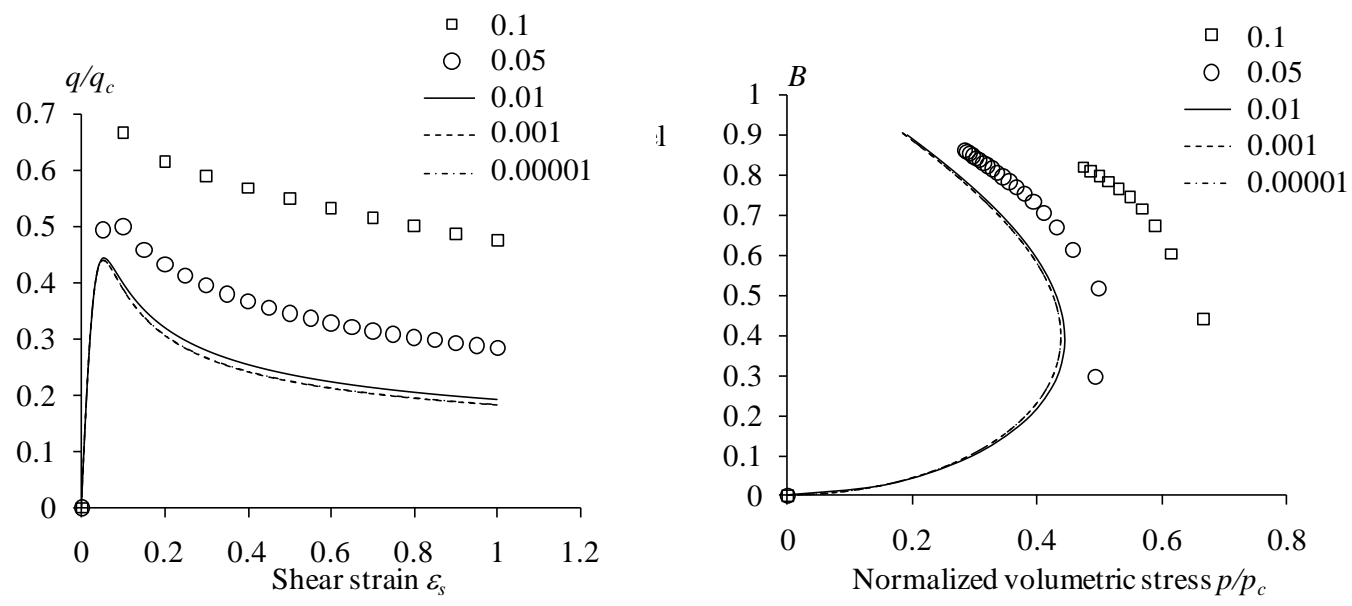

Figure 9 Validation of accuracy in terms of different increment size

\section{Model application}

The behaviour of two typical calcareous soils, Dog's Bay and Chiibishi sands, and Bentheim sandstone are predicted using the above FE model. The consolidated drained triaxial behaviour of these soils are simulated when they are subjected to different initial confining pressures. The single-element model is shown in Figure 2 and boundary conditions in Figure 6. The model's parameters listed in Table 2 are 
mainly from [32], [33] and [34], respectively. The index property $\vartheta$ for Dog's Bay sand can be calculated based on the initial and ultimate cumulative GSDs, while that for Chiibishi sand is determined from the experimentally given initial cumulative GSD, and an assumed fractal ultimate cumulative GSD in equation $F_{u}(x)=\left(x^{3-\alpha}-D_{m}{ }^{3-\alpha}\right) /\left(D_{M}{ }^{3-\alpha}-D_{m}{ }^{3-\alpha}\right)$ where $D_{m}$ and $D_{M}$ denote the minimum and maximum size of grains and the fractal dimension $\alpha$ takes the value $\alpha=2.7$ [35]. The high value, $\vartheta$, for Bentheim sandstone implies very poor initial grading in the test.

Figures 10-11 show the agreement between numerical predictions and experimental observations. From these examples, we can see that the simple breakage model is particulaly suitable for studying crushable granular aggregates, independent of their hardnesses.

Table 2 Model parameters for granular materials

\begin{tabular}{ccccccc}
\hline Crushable sand & $K(\mathrm{MPa})$ & $G(\mathrm{MPa})$ & $p_{\mathrm{c}}(\mathrm{MPa})$ & $M$ & $\omega$ & $\vartheta$ \\
\hline Chiibishi sand & 17.8 & 14.5 & 1.3 & 1.77 & $30^{\circ}$ & 0.2 \\
Dog's bay sand & 25 & 14 & 0.6 & 1.65 & $38^{\circ}$ & 0.65 \\
Bentheim sandstone & 11522 & 7513 & 390 & 1.7 & $70^{\circ}$ & 0.9 \\
\hline
\end{tabular}

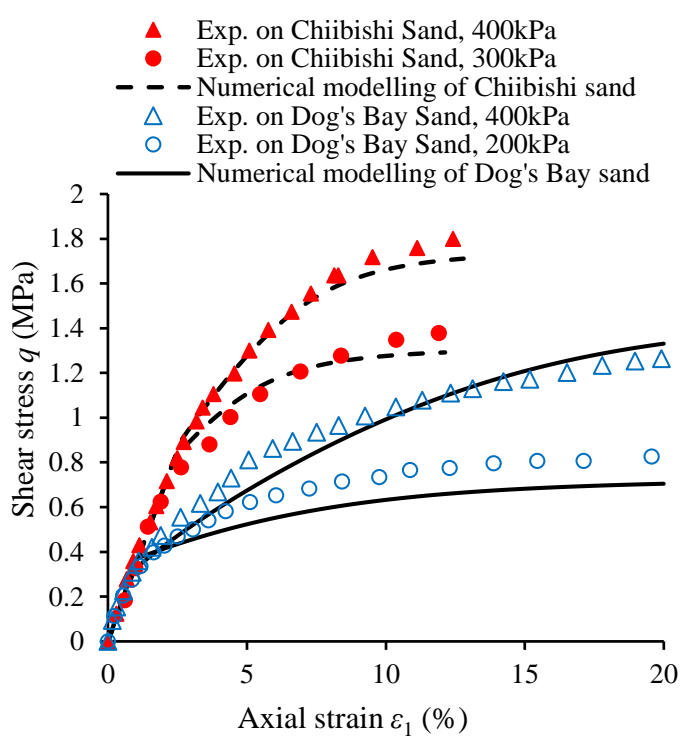

(a)

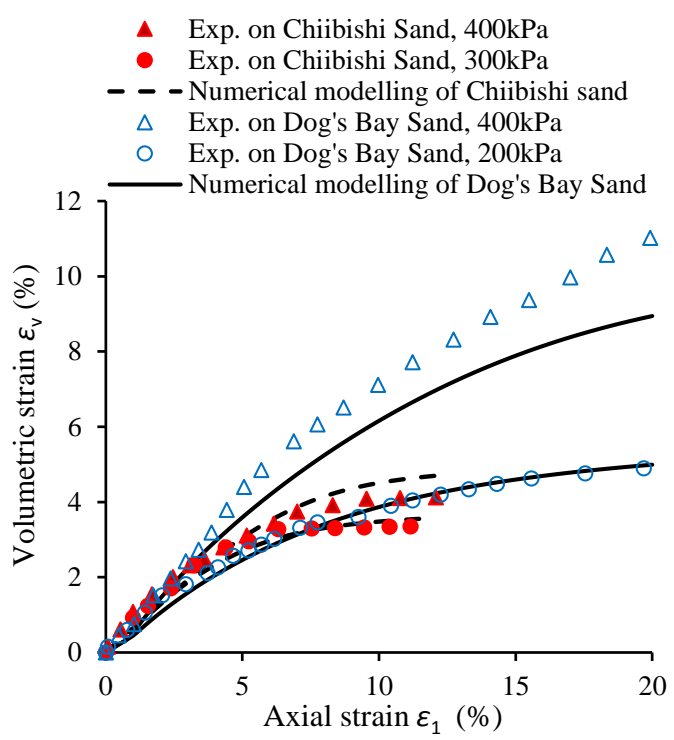

(b)

Figure 10 Drained triaxial shear behaviours of Chiibishi and Dog's Bay sands at different initial confining pressures: (a) shear stress - axial strain and (b) volumetric strain - axial strain (replotted from [26] and the experimental data from [32]). 


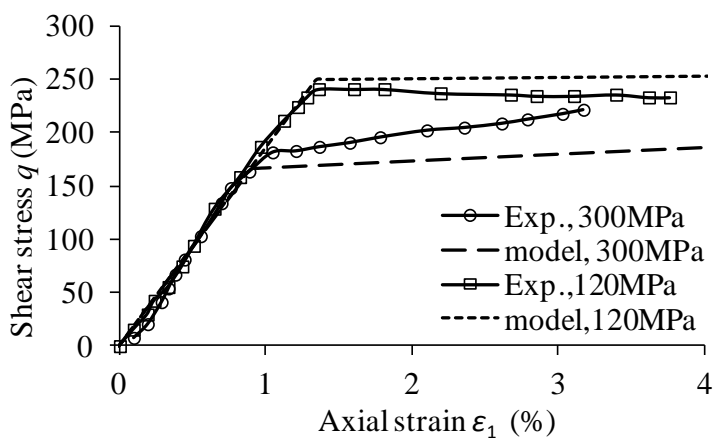

(a)

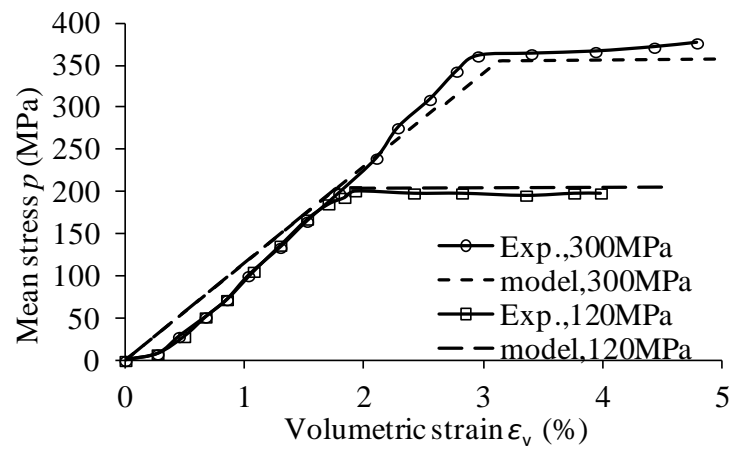

(b)

Figure 11 Drained triaxial shear behaviours of Bentheim sandstone at different initial confining pressures: (a) shear stress - axial strain and (b) mean stress - volumetric strain (experimental data from [34]).

The above numerical examples at the material point level are only used to illustrate the fundamental features of the simple breakage model. Comparatively, with the introduced implementation of the breakage model in various FEAs, some complicated non-uniform BVPs have been numerically studied elsewhere. To avoid any repetition and align with the focus of the paper, these complicated BVPs are not reproduced here. Interested readers are referred to recent papers that discuss each application of the model in detail. For instance, [26] explored the crushing profile around a pile penetrating into crushable sands, while [36] focuses on energy-related crushing phenomenon of minerals passing through a roller mill. Furthermore, an open issue of grain crushing induced compaction localization in porous sandstones has been studied by [23] based on classical discontinuous bifurcation condition. A more recent work by [31] incorporated orientation of localised failure and size effect in the constitutive modelling of the simple breakage model.

\section{Summary}

The recently developed breakage mechanics and the simple breakage model have been discussed, with the focus on how to formulate the mathematical formulations of energy dissipations due to particle breakage, plastic volumetric and shear deformations, and plastic potential. These mathematical details that could have been missed partially or fully in the existing relevant works are elaborated in the present study. This is followed by the detailed illustration of the numerical implementation (with the backward Euler return algorithm) of the model in FEA. Subsequently, 
numerical tests are presented to demonstrate the efficiency of the implementation at the material point level (single element). Next, the key features of the simple breakage model to reflect the mechanical behaviour of granular materials was illustrated by comparing three crushable materials of varying hardness, ranging from very soft (calcareous sands) to very hard (sandstone). More complicated boundary values problems associating with grain crushing could readily be performed with the implemented breakage model, as preliminarily evidenced by [26] on piled foundation and [36] on mineral crushing and on localisation bands [23, 31]. Nevertheless, some other open interesting issues such as nucleation and multi-physical field problems are beyond the capability of the current model, which may require further studies on kinematic behaviours of granular materials.

\section{Acknowledgement}

This research was supported by the State Key Laboratory for GeoMechanics and Deep Underground Engineering, CUMT (Grant No. SKLGDUEK1502). Financial supports from Natural Science Foundation of Jiangsu Province for Distinguished Young Scholars (BK20150005) and National Science Foundation of China (51609072) are also acknowledged. 


\section{Nomenclature list}

\begin{tabular}{|c|c|}
\hline $\begin{array}{l}B \\
D_{i j k l}\end{array}$ & $\begin{array}{l}\text { breakage index } \\
\text { elastic matrix }\end{array}$ \\
\hline$D_{m}$ & minimum grain size \\
\hline$D_{M}$ & maximum grain size \\
\hline$e_{i j}$ & deviatoric strain tensor \\
\hline$E_{B}$ & breakage energy \\
\hline$\overline{E_{B}}$ & dissipative breakage energy \\
\hline$E_{c}$ & breakage energy constant \\
\hline$F(x, B)$ & current cumulative GSD by mass \\
\hline$F_{0}(x)$ & initial cumulative GSD by mass \\
\hline$F_{u}(x)$ & ultimate cumulative GSD by mass \\
\hline$G$ & shear modulus \\
\hline$K$ & bulk modulus \\
\hline$M$ & ratio of failure shear stress to the volumetric one \\
\hline$p$ & mean stress \\
\hline $\bar{p}$ & dissipative mean stress \\
\hline$p_{0}$ & initial mean stress \\
\hline$p_{c}$ & critical isotropic confining pressure \\
\hline$p_{f}$ & mean stress at failure \\
\hline$\underline{q}$ & triaxial shear stress \\
\hline $\bar{q}$ & dissipative triaxial shear stress \\
\hline$q_{f}$ & triaxial shear stress at failure \\
\hline$s_{i j}$ & deviatoric stress tensor \\
\hline$T_{i j m n}$ & tangent stiffness matrix \\
\hline$x$ & grain size \\
\hline$y$ & yield function in mixed stress/breakage space \\
\hline$y^{\text {trial }}$ & yield function at elastic trial point \\
\hline$y^{*}$ & $\begin{array}{l}\text { yield function in generalized dissipative triaxial stress/breakage } \\
\text { space }\end{array}$ \\
\hline$\alpha$ & fractal dimension \\
\hline$\varepsilon_{v}^{e}, \varepsilon_{s}^{e}$ & elastic volumetric and shear strains in triaxial conditions \\
\hline$\varepsilon_{i j}, \varepsilon_{i j}^{e}$ & strain and elastic strain tensors \\
\hline$\varepsilon_{v}, \varepsilon_{s}$ & volumetric and triaxial shear strains \\
\hline$\delta$ & increment \\
\hline$\delta_{i j}$ & Kronecker delta \\
\hline$\delta B$ & incremental breakage \\
\hline$\delta \varepsilon_{v}^{p}, \delta \varepsilon_{s}^{p}$ & incremental plastic volumetric and shear strains \\
\hline$\delta \lambda$ & non-negative plastic multiplier \\
\hline$\vartheta$ & grading index \\
\hline
\end{tabular}




$\begin{array}{ll}\sigma_{i j} & \text { stress tensor } \\ \omega & \text { plastic-breakage coupling angle } \\ \Psi & \text { macroscopic specific elastic strain energy } \\ \delta \Phi & \text { increment of dissipation potential } \\ \delta \Phi_{B} & \text { increment of breakage dissipation } \\ \delta \Phi_{p}^{v} & \text { increment of plastic volumetric dissipation } \\ \delta \Phi_{p}^{s} & \text { increment of plastic shear dissipation }\end{array}$




\section{References}

1. Zhao, J., Sheng, D., Rouainia, M., Sloan, S.W. Explicit stress integration of complex soil models. International Journal for Numerical and Analytical Methods in Geomechanics 2005; 29(12): 1209-1229.

2. Dunne, F., Petrinic, N. Introduction to computational plasticity. Oxford University Press New York. 2005.

3. Sheng, D., Sloan, S.W., Gens, A., Smith, D.W. Finite element formulation and algorithms for unsaturated soils. Part I: Theory. International Journal for Numerical and Analytical Methods in Geomechanics 2003; 27(9): 745-765.

4. Sołowski, W.T., Gallipoli, D. Explicit stress integration with error control for the Barcelona Basic Model: Part I: algorithms formulations. Computers and Geotechnics 2010; 37(1): 59-67.

5. Borja, R.I., Lin, C.-H., Montáns, F.J. Cam-Clay plasticity, Part IV: Implicit integration of anisotropic bounding surface model with nonlinear hyperelasticity and ellipsoidal loading function. Computer methods in applied mechanics and engineering 2001; 190(26): 3293-3323.

6. Simo, J.C., Hughes, T.J. Computational inelasticity. Vol. 7. Springer Science \& Business Media. 2006.

7. Borja, R.I. Cam-Clay plasticity, Part II: Implicit integration of constitutive equation based on a nonlinear elastic stress predictor. Computer Methods in Applied Mechanics and Engineering 1991; 88(2): 225-240.

8. De Borst, R., Crisfield, M.A., Remmers, J.J., Verhoosel, C.V. Nonlinear finite element analysis of solids and structures. John Wiley \& Sons. 2012.

9. Tamagnini, C., Castellanza, R., Nova, R. A generalized backward Euler algorithm for the numerical integration of an isotropic hardening elastoplastic model for mechanical and chemical degradation of bonded geomaterials. International Journal for Numerical and Analytical Methods in Geomechanics 2002; 26(10): 963-1004.

10. Lin, J., Xie, S., Shao, J.F., Kondo, D. A micromechanical modeling of ductile behavior of a porous chalk: Formulation, identification, and validation. International Journal for Numerical and Analytical Methods in Geomechanics 2012; 36(10): 1245-1263.

11. Hoyos, L.R., Arduino, P. Implicit algorithm for modeling unsaturated soil response in three-invariant stress space. International Journal of Geomechanics 2008; 8(4): 266-273.

12. Foster, C., Regueiro, R., Fossum, A., Borja, R. Implicit numerical integration of a three-invariant, isotropic/kinematic hardening cap plasticity model for geomaterials. Computer Methods in Applied Mechanics and Engineering 2005; 194(50): 5109-5138.

13. Amorosi, A., Boldini, D., Germano, V. Implicit integration of a mixed isotropic-kinematic hardening plasticity model for structured clays. International journal for numerical and analytical methods in geomechanics 2008; 32(10): 1173-1203.

14. Zhang, Y., Buscarnera, G. Implicit integration under mixed controls of a breakage model for unsaturated crushable soils. International Journal for Numerical and Analytical Methods in Geomechanics 2015.

15. Collins, I., Houlsby, G. Application of thermomechanical principles to the modelling of geotechnical materials. in Proceedings of the Royal Society of London A: Mathematical, Physical and Engineering Sciences. 1997. The 
Royal Society.

16. Houlsby, G., Puzrin, A. A thermomechanical framework for constitutive models for rate-independent dissipative materials. International Journal of Plasticity 2000; 16(9): 1017-1047.

17. Ziegler, H. An introduction to thermodynamics. North-Holland Publishing Company. 1977.

18. Shao, J.-F., Jia, Y., Kondo, D., Chiarelli, A.-S. A coupled elastoplastic damage model for semi-brittle materials and extension to unsaturated conditions. Mechanics of materials 2006; 38(3): 218-232.

19. Al-Rub, R.K.A., Darabi, M.K. A thermodynamic framework for constitutive modeling of time-and rate-dependent materials. Part I: Theory. International Journal of Plasticity 2012; 34: 61-92.

20. Darabi, M.K., Al-Rub, R.K.A., Masad, E.A., Little, D.N. A thermodynamic framework for constitutive modeling of time-and rate-dependent materials. Part II: Numerical aspects and application to asphalt concrete. International Journal of Plasticity 2012; 35: 67-99.

21. Einav, I. Breakage mechanics - part I: theory. Journal of the Mechanics and Physics of Solids 2007a; 55(6): 1274-1297.

22. Einav, I. Breakage mechanics-Part II: Modelling granular materials. Journal of the Mechanics and Physics of Solids 2007b; 55(6): 1298-1320.

23. Das, A., Nguyen, G.D., Einav, I. Compaction bands due to grain crushing in porous rocks: a theoretical approach based on breakage mechanics. Journal of Geophysical Research: Solid Earth 2011; 116(B8).

24. Nguyen, G.D., Einav, I., The energetics of cataclasis based on breakage mechanics, in Mechanics, Structure and Evolution of Fault Zones. 2009, Springer. p. 1693-1724.

25. Tengattini, A., Das, A., Nguyen, G.D., Viggiani, G., Hall, S.A., Einav, I. A thermomechanical constitutive model for cemented granular materials with quantifiable internal variables. Part I-theory. Journal of the Mechanics and Physics of Solids 2014; 70: 281-296.

26. Zhang, C., Nguyen, G., Einav, I. The end-bearing capacity of piles penetrating into crushable soils. Géotechnique 2013; 63(5): 341.

27. Buscarnera, G., Einav, I. The yielding of brittle unsaturated granular soils. Géotechnique 2012; 62(2): 147.

28. Rubin, M., Einav, I. A large deformation breakage model of granular materials including porosity and inelastic distortional deformation rate. International Journal of Engineering Science 2011; 49(10): 1151-1169.

29. Einav, I. Soil mechanics: breaking ground. Philosophical Transactions of the Royal Society of London A: Mathematical, Physical and Engineering Sciences 2007d; 365(1861): 2985-3002.

30. Einav, I. Fracture propagation in brittle granular matter. in Proceedings of the Royal Society of London A: Mathematical, Physical and Engineering Sciences. 2007c. The Royal Society.

31. Nguyen, G.D., Nguyen, C.T., Bui, H.H., Nguyen, V.P. Constitutive modelling of compaction localisation in porous sandstones. International Journal of Rock Mechanics and Mining Sciences 2016; 83: 57-72.

32. Kuwajima, K., Hyodo, M., Hyde, A.F. Pile bearing capacity factors and soil crushabiity. Journal of geotechnical and geoenvironmental engineering 2009; 135(7): 901-913.

33. Coop, M., Sorensen, K., Freitas, T.B., Georgoutsos, G. Particle breakage 
during shearing of a carbonate sand. Géotechnique 2004; 54(3): 157-164.

34. Baud, P., Klein, E., Wong, T.-f. Compaction localization in porous sandstones: spatial evolution of damage and acoustic emission activity. Journal of Structural Geology 2004; 26(4): 603-624.

35. Turcotte, D. Fractals and fragmentation. Journal of Geophysical Research: Solid Earth 1986; 91(B2): 1921-1926.

36. Zhang, C., Nguyen, G.D., Kodikara, J. An application of breakage mechanics for predicting energy-size reduction relationships in comminution. Powder Technology 2016; 287: 121-130. 